\title{
Is Burn-Out the Main Issue? Impact of Aggressiveness and Depression in Burn-Out Among Operators in Oncology
}

\author{
Romeo Marco \\ Università Politecnica delle Marche, Ancona, Italy; \\ Fondazione Rossetti Fedecostante, Ancona, Italy
}

\author{
Anna Vespa, Maria Velia Giulietti \\ INRCA-IRCCS-National Institute of Science and Health for \\ Aging, Ancona, Italy
}

\author{
Riccardo Giampieri, Tania Meletani, Ilaria Fiordoliva, Maria Giuditta Baleani, Sofia Formentini, \\ Rossana Berardi \\ Università Politecnica delle Marche, Ancona, Italy
}

\begin{abstract}
Over the last 30 years, Burn-out Syndrome has been mainly matched to psychological discomfort among the staff in Oncology Departments, therefore, it has been considered as the unique issue to be diagnosed, treated, and prevented. Our study is aimed to evaluate the relation between psychological states and traits with different Burn-out Scales from a clinical point of view. Three questionnaires: Link Burn-out Questionnaire (LBQ), State-Trait Anger Expression Inventory-2 (STAXI-2), and Beck Depression Inventory (BDI) have been given to the working staff including Medical Oncologists, Specialist Registrant, Nurses, and Healthcare Assistants at the Oncology Department from February to April 2016. Seventy-two operators have been included in our analysis. Male/Female (M/F) ratio was 18/54. Median age was 37 years old (range from 20 years old to 62 years old). We did not show a significant correlation between depression and operators' age, years of work, professional role, and relational decline, while a statistically significant association was observed between depression and professional ineffectiveness $(\mathrm{p}=0.042)$, disillusion $(\mathrm{p}=0.0003)$, and psychophysical exhaustion $(\mathrm{p}=0.00001)$. According to STAXI 2, $15 \%$ of the personnel had a high expression of aggressiveness while $10 \%$ was over-controlled. Aggressiveness was statistically related to depression $(\mathrm{p}=0.001)$, disillusion $(\mathrm{p}=0.009)$, and psychophysical exhaustion $(\mathrm{p}=0.012)$. This study showed that aggressiveness and depression play a crucial role in psychological discomfort in an Oncology Department operators. Therefore, they should be taken into account together with burn-out when performing screening procedures for the psychological discomfort in the same setting.
\end{abstract}

Keywords: aggressiveness, anxiety, burn out, cancer, depression, oncology

\footnotetext{
*Acknowledgements: Funding: this work was supported by Fondazione Rossetti Fedecostante, via Mantegna 1, 60019 Senigallia (Italy). Conflict of Interest: The authors declare that they have no conflict of interest.

Romeo Marco, MD, Clinica Oncologica, Università Politecnica delle Marche; Fondazione Rossetti Fedecostante. Anna Vespa, MD, Department of Neurology, INRCA-IRCCS-National Institute of Science and Health for Aging. Maria Velia Giulietti, MD, Department of Neurology, INRCA-IRCCS-National Institute of Science and Health for Aging. Riccardo Giampieri, Ph.D., MD, Medical Oncology Unit, Università Politecnica delle Marche.

Tania Meletani, MD, Medical Oncology Unit, Università Politecnica delle Marche. Ilaria Fiordoliva, MD, Medical Oncology Unit, Università Politecnica delle Marche. Maria Giuditta Baleani, MD, Medical Oncology Unit, Università Politecnica delle Marche. Sofia Formentini, MD, Medical Oncology Unit, Università Politecnica delle Marche. Rossana Berardi, MD, Medical Oncology Unit, Università Politecnica delle Marche.
} 


\section{Background}

Burn out syndrome was defined by C. Maslach during the 1970s as a pathological process characterized by emotional exhaustion, depersonalization, and reduced personal accomplishment, which affects individuals who work with other people including health professions' workers (Maslach \& Jackson, 1981). This condition is a negative response to chronic stress and leads to deteriorating work performance and relation with patients. It has a high incidence among cancer department workers, in relation to specific features regarding disease history, patients and operators responses (Gómez-Urquiza et al., 2016; Trufelli et al., 2008). Well-known risk factors include continuous contact with death, conflicts with colleagues, lack of recognition of achievements, and work overload. Moreover, cultural aspects, such as high expectations of professional success, insufficient psychological preparation, and personality traits characterized by low self-esteem, feelings of inadequacy, social anxiety, and withdrawal from others increase the risk of developing burn out syndrome (McCraine \& Brandsma, 1988; Maslach, Schaufeli, \& Leiter, 2001). Burn out can be characterized by clinical and psychological symptoms: weakness, drowsiness, headache, back pain, nausea, diarrhea, dizziness, cynicism, sense of frustration, reduced interest in their work, negative reactions towards family and colleagues, sense of guilt, apathy, family conflicts, and alcohol or drugs abuse. Definition of burn out by Maslach represented the basis of Maslach Burnout Inventory (MBI), which is the most common tool to detect burn out. The questionnaire includes 22 items (divided into three subscales: nine items for personal exhaustion, five for depersonalization, and eight for personal accomplishment). The respondent has to give a score ranging from zero, that means "never" to six, that means "every day". The result consists of three separated scores, corresponding to each component of burn out syndrome. Some alternate versions of the survey had been developed, one for educators and teachers (MBI Educators Survey) and another one for workers in other occupations (MBI General Survey), therefore the original version made for professionals in the human services has been named MBI Human Services Survey. Several translated versions had been validated in different countries (Maslach, Schaufeli, \& Leiter, 2001; Enzmann, Schaufeli, \& Girault, 1995). The evaluation of Burn Out may also be conducted through the Link Burn Out Questionnaire, an evolution of Maslach Burn-out Inventory. This scale has been validated in different fields included medical and social worker fields (Murdaca, Oliva, \& Nuzzaci, 2014).

Depression is partially related to burn out. In fact, it may present with similar symptoms and may co-exist in the same person (Aholaa, Hakanena, Perhoniemia, \& Mutanen, 2014). Beck Depression Inventory (BDI) was developed during the 1960s and subsequently modified in 1996 as Beck Depression Inventory-Second Edition (BDI-II) according to the definition for Major Depression.

The BDI-II consists of 21 items scored from zero to three, with increasing scores indicating greater depression severity and it has been validated in heterogeneous samples (Beck, Ward, Mendelson, Mock, \& Erbaugh, 1961; Beck, Steer, \& Brown, 1996; Whisman, Perez, \& Ramel, 2000). The aim of this study is to evaluate the impact of burn out on the operators' quality of life in oncology field and to screen the personnel (including physicians and nurses) working in our institution in order to design paths for training and psychological support. Co-primary aim of our analysis is to assess correlations between scales used to measure different forms of psychological distress. We administered the Link Burn-out Questionnaire (LBQ), State-Trait Anger Expression Inventory-2 (STAXI-2) that evaluates the aggression manifestation (internal aggression plus external aggression, and plus self and external control), and Beck Depression Inventory (BDI) to measure depression. 


\section{Methods}

We administered the LBQ, STAXI-2 and BDI to all the staff (medical oncologists, fellows of the Postgraduate School of Medical Oncology, nurses, and healthcare assistants) working at Ancona Hospital, from February to April 2016.

LBQ assesses burn-out through 24 items grouped in four dimensions: Psycho-physical exhaustion, Detriment of the relationships, Professional Inefficacy and Disillusion. Each dimension had eight items: four positive and four negative.

Each question can be answered using by six points: "never" (the 1st point), "rarely" (the 2nd point), "once or more times a month" (the 3rd point), "every week" (the 4th point), "more than once a week" (the 5th point), and "every day" (the 6th point).

The sum of the items was assigned as follows: 1-2 score identifies subjects with a positive perception of their working condition; 3-7 score identifies subjects with worse perception of their working condition; and 8-9 score identifies subjects who have high risk to develop burnout syndrome.

Even though there is a general lack of data regarding its use in medical settings, LBQ scale was chosen due to the teaching nature of our University Hospital, therefore, it was judged more appropriate compared to standard MBI.

STAXI-2 measures the intensity of anger as an emotional state and the disposition to experience angry feelings as a personality trait trough six scales (State anger, Trait anger, Anger expression-out, Anger expression-in, Anger control-out, and Anger control-in) composed by 57 items. In our study, we used 44-48 as range to identify the anger.

BDI is designed for individuals aged 13 and older, and measures depression trough a 21 multiple-choice questions self-report inventory. It uses ranked responses ranging from zero to three to assess variations in the severity of depression over time.

The sum of the score allows to identify mild depression ( $\geq 13$ points), moderate depression (20-29 points), and severe depression ( $\geq 30$ points).

Due to the cross-sectional observational nature of the analysis, no pre-planned sample size was calculated before study conduction. Statistical analysis was performed with MedCalc Statistical Software version 14.10.2. The association between categorical variables was estimated by Fisher's exact test for categorical binomial variables or by chi-square test when indicated. Level of statistical significance $p$ was set at 0.05 for all conducted analyses. Tested variables included gender (male vs female), age, years of work, professional role, and family status (celibacy, married, divorced, etc.).

Ethical approval was not required since the procedures included into the study were according to standard clinical practice, but informed consent was obtained from all individual participants included in the study. All procedures performed in studies, involving human participants, were in accordance with the ethical standards of the institutional and/or national research committee and with the 1964 Helsinki declaration and its later amendments or comparable ethical standards.

\section{Results}

Seventy-two operators have been included in our analysis, Male/Female (M/F) ratio was 18/54 and 37 years old (range from 20 years old to 62 years old). In this study, the Nurses were the most representative 
professional role, followed by post-graduate Oncology workers and trainees in Medical Oncology (Table 1).

LBQ scale showed that only five operators (7\%) had high risk to develop burn out syndrome: three operators because of relational deterioration and two operators owing to disillusion. In particular, 28 operators (39\%) showed a risk to develop relational deterioration: three of them (4\%) had high risk and 25 (35\%) showed a moderate risk to develop relational deterioration. Moreover, two operators $(3 \%)$ had high risk to develop disillusion. Psychophysical exhaustion was observed in $21 \%$ of the group.

BDI scale showed that eight operators (11\%) presented depression (BDI score $\geq 13$ ).

Forty-one operators (57\%) complained sleep problems, $47 \%$ of the sample reported (34 operators) fatigue, 32 operators (44\%) had agitation, 24 operators (33\%) self criticism, and 23 operators (32\%) had loss of sex drive.

STAXI-2 showed that 11 operators had high expression of aggressiveness, in particular five operators had a score $>48$ that means high aggressiveness expression and six operators had a score 44-48 that means mild aggressiveness expression. Furthermore, seven operators (10\%) showed over-control.

In our analysis, depression was not related to operators' age, years of work, professional role, and relational decline, while a statistically significant association was observed between depression and professional ineffectiveness $(\mathrm{p}=0.042)$, disillusion $(\mathrm{p}=0.0003)$, and psychophysical exhaustion $(\mathrm{p}=0.00001)$.

Aggressiveness was statistically related to depression $(p=0.001)$, disillusion $(p=0.009)$, and psychophysical exhaustion $(\mathrm{p}=0.012)$ (Table 2).

Table 1

Study Sample Stratified by Professional Role

\begin{tabular}{ll}
\hline Operators & n. $(\%)$ \\
\hline Specialist registral & $15(21)$ \\
Specialist in oncology & $17(23.5)$ \\
Nurses & $23(31.9)$ \\
Nursestrainee & $5(6.9)$ \\
Healthcare assistants & $12(16.7)$ \\
Total & $72(100)$ \\
\hline
\end{tabular}

Table 2

Correlation About LBQ, BDI and STAXI II Scales. Level of Statistical Significance P Was Set at 0.05 for All Conducted Analyses

\begin{tabular}{|c|c|c|c|c|}
\hline & Relational decline & $\begin{array}{l}\text { Professional } \\
\text { ineffectiveness }\end{array}$ & Disillusion & $\begin{array}{l}\text { Psychophysical } \\
\text { exhaustion }\end{array}$ \\
\hline $\begin{array}{l}\text { Years of work } \\
(\leq 5 \text { vs } \geq 5)\end{array}$ & $\mathrm{P}=0.050$ & $\mathrm{P}=1.000$ & $P=0.096$ & $P=0.022$ \\
\hline Age & $P=0.621$ & $P=0.266$ & $\mathrm{P}=0.506$ & $P=0.771$ \\
\hline Gender & $P=0.780$ & $P=0.681$ & $\mathrm{P}=1.000$ & $\mathrm{P}=1.000$ \\
\hline Depression & $\mathrm{P}=0.265$ & $\mathrm{P}=0.042$ & $\mathrm{P}=0.0003$ & $\mathrm{P}=0.00001$ \\
\hline $\begin{array}{l}\text { Aggressiveness } \\
\text { Expression }\end{array}$ & $P=0.319$ & $P=0.117$ & $P=0.009$ & $\mathrm{P}=0.012$ \\
\hline
\end{tabular}

\section{Discussion}

The Burn out syndrome is an emerging issue in oncology operators. Nevertheless, the MBI does not assess the psychological discomfort in all exhaustive manner. 
This study confirmed this statement showing that Burn Out cannot be considered the unique tool to measure the psychological disorder, but should be integrated with other scales, including STAXI-2 and BDI.

Our study also tried to assess the impact of other variables which were not strictly related to work, such as cultural and familiar problems, and personal discomfort, as potential confounders in the evaluation of causes of psychological distress in this field.

Moreover, our data showed that aggressiveness and depression play an essential role and need to be taken into account together with burn-out when screening the psychological discomfort among operators in Oncology.

Similarly to our analysis, Muscatello et al. evaluated the correlation between MBI and STAXI: anger was correlated to Burn Out and appeared to be an understudied emotion if compared to other emotional states such as anxiety and depression (Muscatello et al., 2006).

According to our study, LBQ might be much more reliable in assessing psychosocial discomfort compared to Maslach Burn Out inventory. In particular, even though we demonstrated a significant correlation between LBQ, BDI, and STAXI-2, different correlations were observed when we evaluated separately the two scales of LBQ, one related to detriment of relationships and another one related to professional inefficacy: detrimental relationships was not correlated to depression (measured by BDI) whereas the opposite appeared evident when professional inefficacy was assessed. Maslach Burn Out inventory makes no difference between detriment of relationships and professional inefficacy, thus losing this meaningful differences.

Several studies already proved that depression is an important risk factor in oncology field (Whippen \& Canellos, 1991; Medisauskaite \& Kamau, 2017). Prevalence of oncologists in distress: Systematic review and meta-analysis analyzed 1713 nurses with MBI and the Shortened Beck Depression Questionnaire, respectively. Somatization was measured by the Patient Health Questionnaire. This study showed depressive symptoms and clinical depression in 35\% and $13 \%$ subjects, respectively, emotional exhaustion in $44 \%$, depersonalization in $36 \%$, and decreased personal accomplishment in 74\% (Ádám, Cserháti, \& Mészáros, 2015).

Another pilot study evaluated 30 primary care clinicians using MBI at baseline and one day, eight weeks, and nine months post psychological intervention underlining the need of mindfulness training. In fact, psychological intervention led to reduced burn-out risk, depression, anxiety, and stress. Modified mindfulness training may be a time-efficient tool to help support clinician health and may have implications for patient care (Fortney, Luchterhand, Zakletskaia, Zgierska, \& Rakel, 2013).

Even though we suggested mindfulness training for the operators, it should also be considered that workers' behavior is influenced also by their professional role, family status, stress-factors, and patient relationships (Allen, Holland, \& Reynolds, 2015; Zoni, Lucchini, \& Alessio, 2010).

\section{Conclusions}

The management of Burn out should consider the whole psychological dimension of the operators through the use of different scales.

This study showed that aggressiveness and depression play a crucial role in psychological discomfort in an Oncology Department operators. Therefore, they should be taken into account together with burn-out when performing screening procedures for the psychological discomfort in this setting. Moreover, our results underlined the need of educational programs and preventive interventions to control burnout.

Due to the relatively small sample size, our data need to be confirmed in further investigations in larger number of operators and hopefully in a multi-institutional setting. 
Future aim will be to evaluate improvements in psychological discomfort in the same sample group after psycho-oncologist intervention.

\section{References}

Ádám, S., Cserháti, Z., \& Mészáros, V. (2015). High prevalence of burnout and depression may increase the incidence of comorbidities among Hungarian nurses. Ideggyogy Sz, 68(9-10), 301-309.

Aholaa, K., Hakanena, J., Perhoniemia, R., \& Mutanen, P. (2014). Relationship between burnout and depressive symptoms: A study using the person-centred approach. Burnout Research, 1(1), 29-37.

Allen, B. C., Holland, P., \& Reynolds, R. (2015). The effect of bullying on burnout in nurses: The moderating role of psychological detachment. J Adv Nurs, 71(2), 381-390. doi: 10.1111/jan.12489

Beck, A. T., Steer, R., \& Brown, G. K. (1996). Beck Depression Inventory-Second Edition manual. San Antonio, TX: The Psychological Corporation.

Beck, A. T., Ward, C. H., Mendelson, M., Mock, J., \& Erbaugh, J. (1961). An inventory for measuring depression. Arch Gen Psychiatry, 4, 561-571.

Enzmann, D., Schaufeli, W. B., \& Girault, M. (1995). The validity of the Maslach Burnout Inventory in three national samples. In L. Bennett, D. Miller, \& M. Ross (Eds.), Health workers and AIDS: Research, intervention and current issues in burnout and response (pp. 131-150). Chur: Harwood Academic Press.

Fortney, L., Luchterhand, C., Zakletskaia, L., Zgierska, A., \& Rakel, D. (2013). Abbreviated mindfulness intervention for job satisfaction, quality of life, and compassion in primary care clinicians: A pilot study. Ann Fam Med, 11(5), 412-420. doi: 10.1370/afm. 1511

Gómez-Urquiza, J. L., Aneas-López, A. B., Fuente-Solana, E. I., Albendín-García, L., Díaz-Rodríguez, L., \& Fuente, G. A. (2016). Prevalence, risk factors, and levels of burnout among oncology nurses: A systematic review. Oncol Nurs Forum 1, 43(3), 104-120.

Maslach, C., \& Jackson, S. E. (1981). MBI: Maslach Burnout Inventory. Consulting Psychologists Press. California, CA: Palo Alto.

Maslach, C., Schaufeli, W. B., \& Leiter, M. P. (2001). Job burnout. Annu Rev Psychol, 52, 397-422.

McCraine, E. W., \& Brandsma, J. M. (1988). Personality antecedents of burnout among middle-aged physicians. Behav Med, 14(1), 30-36.

Medisauskaite, A., \& Kamau, C. (2017). Prevalence of oncologists in distress: Systematic review and meta-analysis. Psychooncology Jan 23. doi: 10.1002/pon.4382

Murdaca, A. M., Oliva, P., \& Nuzzaci, A. (2014). Fattori individuali e contestuali del burnout: una ricerca descrittiva sugli insegnanti curricolari e di sostegno. Giornale Italiano della Ricerca Educativa-Italian Journal of Educational Research (C) Pensa MultiMedia Editore srl, special issue, 99-120. ISSN 2038-9736 (in press)/ISSN 2038-9744 (on line).

Muscatello, M. R., Bruno, A., Carroccio, C., Cedro, C., La Torre, D., Di Rosa, A. E., ... di Orio, F. (2006). Association between burnout and anger in oncology versus ophthalmology health care professionals. Psychol Rep, 99(2), 641-650.

Trufelli, D. C., Bensi, C. G., Garcia, J. B., Narahara, J. L., Abrão, M. N., Diniz, R. W., .. Del Giglio, A. (2008). Burnout in cancer professionals: A systematic review and meta-analysis. Eur J Cancer Care, 17(6), 524-531.

Whippen, D. A., \& Canellos, G. P. (1991). Burnout syndrome in the practice of oncology: Results of a random survey of 1000 oncologist. J Clin Oncol, 9,1916-1920.

Whisman, M. A., Perez, J. E., \& Ramel, W. (2000). Factor structure of the Beck Depression Inventory-Second Edition (BDI-II) in a student sample. Clin Psychol, 56, 545-551.

Zoni, S., Lucchini, R., \& Alessio, L. (2010). Integration of subjective and objective methods for stress related risks evaluation in the health care sector. G Ital Med Lav Ergon, 32(3), 332-336. 